\title{
A translation-based generation effect in bilingual recall and recognition
}

\author{
WARD O'NEILL, LISE ROY, and ROGER TREMBLAY \\ Université de Moncton, Moncton, New Brunswick, Canada
}

\begin{abstract}
Two experiments were performed with incidental study procedures in which generation effects were observed for relatively balanced French-English bilingual subjects on measures of both free recall and recognition. Experiment 1 used a within-subject design, and Experiment 2 used a between-subject design. In both experiments, reading translations and reading same-language repetitions generally resulted in similar amounts of retention. Experiment 2 and Experiment 3 also showed that the generation effect was abolished under intentional study procedures because of an enhancement of reading-based retention. The results suggested that previous failures to obtain a translation-based generation effect may have been due to the use of intentional study procedures. The pattern of effects was explained in terms of a single-code model of bilingual linguistic representation and an individual-item processing interpretation of the generation effect.
\end{abstract}

A number of studies inspired by the dual-coding framework of bilingual memory (Paivio, 1986; Paivio \& Desrochers, 1980) have compared the effects of translationbased encodings with the effects of both simple copying and image-based encodings in determining retention in bilingual subjects (Arnedt \& Gentile, 1986; Paivio \& Lambert, 1981). In these studies, subjects either copied, translated, or generated images to stimulus words. The results showed that translation promoted greater free recall than did the copying of individual words but less free recall than did imagery-based encodings. It has been demonstrated further that translation-based encodings lead to higher levels of recall than do within-language synonymbased encodings (Vaid, 1988). These results have been taken as support for the hypothesis that bilinguals possess two language-specific verbal representational systems, corresponding to each of their two languages. This is the basic assumption of the dual-coding theory of bilingual memory (Paivio, 1986; Paivio, Clark, \& Lambert, 1988; Paivio \& Desrochers, 1980).

The theory further argues that because of their greater specificity, cross-language connections (primarily translations) are generally stronger than within-language connections (e.g., synonyms), which are considered to possess greater variability. The theory thus argues that bilingual repetitions (translations), whether based on generation, as in the above studies, or on reading, will lead to better recall than will identical repetitions. This will occur because bilingual repetitions occasion the stimulation of distinct traces in each verbal system, whereas identical repetitions occasion the stimulation of redundant traces within the same verbal system. Given that the connections

This research was supported in part by a grant from the Conseil de Recherche, Université de Moncton, to the first author. Correspondence should be addressed to W. O'Neill, Department of Psychology. University of Moncton, Moncton, NB, Canada E1A $3 E 9$. between traces based on translations are viewed as stronger than the within-language connections, the theory is also consistent with the finding that translations promote greater recall than do synonyms.

Slamecka and Katsaiti (1987) have pointed out that the translation-based encoding results could be interpreted as an example of the generation effect. Generation effects have usually been defined as being present when greater retention is found for target words generated to cue words in conformity with certain rules (e.g., synonyms, antonyms, associates, etc.) applied to partial word information than when the target words are actually presented for study (Slamecka \& Graf, 1978; Slamecka \& Katsaiti, 1987). Thus, the results of the translation-based encoding studies would not require the postulation of languagespecific verbal representation systems but could be explained in terms of generation processing similar to that occurring with unilingual cue-target relationships.

However, Slamecka and Katsaiti (1987) also pointed out a serious control problem in the translation-based encoding studies that may have accounted for much of the superiority of translation-based encoding over copying. For items in the translate condition, two paths existed that could have led to successful recall. Subjects could have directly recalled the target (generated word) or inferred the target from recall of the cue word and the subsequent translation of it. In contrast, under the control procedures employed, only direct recall of the copied word would have led to correct responses. Although this may be interpreted as a restatement of dual-coding theory in processing terms, it would appear to render redundant the postulation of language-specific representational systems. A single representational system model (Liepmann \& Saegert, 1974; MacLeod, 1976; Potter, So, von Eckardt, \& Feldman, 1984; Schwanenflugel \& Rey, 1986) allowing for dual access by means of language-specific input processes to a common semantic representational system 
would be equally compatible with the processing-paths explanation.

A similar criticism can be made of the comparison between encodings based on translations and those based on synonym production. If it is assumed that translation equivalents are more strongly associated than are synonyms (Dalrymple-Alford, 1985; Kolers \& Gonzalez, 1980; Paivio et al., 1988), recall of the cue words may lead to more accurate inferences of the targets for generated translations than for synonyms. These more accurate inferences would lead to greater recall for the translations, obviating the need for a language-specific semantic-stores hypothesis.

When Slamecka and Katsaiti (1987, Experiments 1 and 2) compared standard generation-effect procedures, using either cross-language synonyms or antonyms to a crosslanguage control procedure in which subjects read both the cue word and its other language synonym or antonym, no evidence was found for a generation effect in either between- or within-list designs. Similarly, Durgunoglu and Roediger (1987), using a within-list procedure, failed to find a statistically significant difference between translation generation and translation reading on measures of recall, recognition, or word-fragment completion. ${ }^{1}$

The results of these studies would seem to indicate that cross-language processes, such as translation, do not produce generation effects when compared with appropriate cross-language control procedures. But this finding does not necessarily contradict bilingual dual-coding theory. The theory argues that translation is superior to copying because both of the bilingual's linguistic coding systems are activated during translation, whereas in the withinlanguage copying or reading conditions, only one coding system is activated. The control procedure of asking subjects to read or copy the translation equivalents would have activated both coding systems and consequently resulted in an outcome similar to that obtained with generated translations. In accord with this idea, it has been reported that with continuous serial presentations of word lists, a free-recall advantage exists for translations as opposed to same-language repetitions when short lags are employed (Glanzer \& Duarte, 1971; Paivio et al., 1988). Durgunoglu and Roediger (1987) also observed significantly greater free recall and recognition for the read-translation conditions than for the read-same-language-repetitions conditions.

The absence of the translation-based generation effect may be taken as strong evidence for the languagespecificity of the bilingual's verbal representation systems because it contrasts markedly with the numerous withinlanguage encoding procedures employing various types of orthographic, acoustic, and semantic word relationships that promote generation effects (Hirshman \& Bjork, 1988; Slamecka \& Katsaiti, 1987). Because translation does not lead to the same outcome as do many within-language connective processes, it would appear difficult to defend a single-code view of bilingual verbal representation.
However, both of the failures to obtain a cross-language generation effect may have been caused by methodological problems. Slamecka and Katsaiti (1987, Experiments 1 and 2) used Greek-English bilinguals who "were sufficiently fluent in both the Greek and the English languages to be able to accomplish the assigned tasks without any difficulty"' (p. 591). No additional indication was provided as to the degree of the subjects' functional bilingualism or of the relative dominance of one or the other of their two languages. It is possible that the subjects were basically English speakers with a knowledge of Greek vocabulary but whose bilingual information-processing abilities were relatively poorly developed. The present experiments employed French-English bilinguals with relatively balanced abilities and considerable functional experience in their languages.

The subjects in the Durgunoglu and Roediger (1987) study were relatively balanced Spanish-English bilinguals, but the nature of the control procedure may have encouraged subjects to generate translations in their readEnglish-and-Spanish-translation conditions. Conditions were blocked so that subjects received the instruction to read both Spanish and English translations prior to the presentation of the pairs of words constituting this condition. Subjects viewed the Spanish word on a screen and subsequently read the English word in a booklet. Thus subjects might, on occasion, have generated the English translation prior to actually reading the English word, making the control condition similar to the translation condition and masking any potential generation effects. In the present study, an attempt was made to reduce the likelihood of spontaneous translation in the control condition by presenting the translations simultaneously.

Another possible problem in both of the above studies is that subjects were informed prior to the study phases that memory tests would follow. Such intentional learning procedures allow for the adoption of learning strategies that might mask the presence of generation effects. Experiment 2 of the present study includes a comparison of both incidental and intentional procedures in order to evaluate this possibility.

The purpose of Experiment 1 was to determine whether a robust translation-based generation effect can be obtained when linguistically balanced bilingual subjects study lists under incidental learning conditions. Experiment 1 compared three study conditions on measures of free recall and recognition. The first condition required subjects to generate translations, the second condition required them to read translations, and the third condition required subjects to read same-language repetitions. Dual-coding theory would predict that the read-translation condition should promote greater retention than should the readrepetitions condition. A single-code theory would not predict such a difference (because translations are functionally equivalent to repetitions of the same word) but would predict the presence of a translation-based generation effect. 


\section{EXPERIMENT 1}

\section{Method}

\section{Subjects}

The subjects were 42 French-English bilinguals who participated voluntarily. They were selected from a pool composed of approximately 300 psychology undergraduates who had completed a selfrating language test (Macnamara, 1967). The test consisted of 5 -point scales of reading, writing, speaking, and comprehension skills in both languages. Only subjects who responded with at least the three highest ratings (corresponding to average, above average, and very above average) were selected. Respondents with deviations greater than two units between their self-evaluations in the two languages on any one scale were eliminated. All of the subjects reported French as their maternal, preferred, and most frequently used language, but all of them currently employed English at least three times a week, on average, in their daily affairs.

\section{Materials}

We obtained 120 words from the Vikis-Freibergs (1974) French frequency norms. Words were initially selected so that their most common English translations were orthographically and acoustically distinct from them. The French words, along with the first letters of their most common English translations, were presented to 5 fluent bilingual judges who were asked to generate the translations. Only those words correctly translated by all of the judges were retained. This procedure was repeated for the English words using 5 new judges. Once again, only those words for which all judges generated the correct translations were retained.

Half of the retained words served as cues and targets in the study conditions, and the other half were employed as distractors in the recognition test. The cue and target words were blocked into three sets of 20 French-English pairs. Each set occurred once within each study condition in the English target conditions, and each occurred once for each study condition in the French target conditions but with the English and French pairs reversed. When a set occurred in the read-same-language condition, the target was simply a repetition of the cue.

Six different booklets were prepared, each consisting of 63 pages containing either a word and its translation, a word and its repetition, or a word and the first letter of its translation followed by dashes corresponding to the number of letters in the translation. Cues and targets were presented in a single horizontal line separated by a dash in the middle of the page. Before the subjects were given each set of 20 pairs, they viewed an instruction sheet identifying the subsequent study condition. The order of study conditions was counterbalanced across booklets within each language condition.

Two recognition lists were constructed in both English and French. In all cases, they consisted of the 60 targets randomly intermixed with 60 distractor words.

\section{Procedure and Design}

The experiment contained a between-subject variable (language of targets) and a within-subject variable (study conditions). Half of the subjects received English words as cues followed by either their repetition, their French translation, or the first letter of their translation. This defined the French target group, whereas the reverse arrangement defined the English target group.

Three study conditions were employed. In one condition, the subjects viewed repetitions and were asked to read the word twice and copy the word (repetition). In a second condition, the subjects viewed translation equivalents and were asked to read the words and copy the second word (translation). In the third condition, the subjects viewed a word and the first letter of its translation and were asked to read the word and write the translation (generation).

Each set of words appeared the same number of times in each of the three possible positions in the booklets. Thus, three differ- ent English and three different French booklets were produced. Seven subjects received each version of the booklets.

The subjects were tested in groups of between 3 and 8 . Upon arrival in the test room, they were instructed, in French, as to the nature of the experimental task but were not informed that retention tests were to follow. The booklets were then given to the subjects. The experimenter told the subjects to write the target word in the booklet and turn to the next page when signaled. Eight seconds were allowed for each response. Between blocks of study conditions, $30 \mathrm{sec}$ were provided, during which the subject was reminded of and read the instructions for the next study condition. Following completion of the study phase, the booklets were collected and the subjects were given $5 \mathrm{~min}$ in which to complete a language-history questionnaire. The subjects were then given a blank sheet of paper and allotted $10 \mathrm{~min}$ to recall the target words in any order. The experimenter emphasized the fact that the targets should be the only words to be recalled. Following this, the subjects received the relevant recognition list and were asked to indicate, for each word, if the word had been or had not been on the study list.

\section{Results}

Only three errors of target generation occurred, and these items were eliminated from the subsequent analyses. The proportion of correctly recalled words was calculated on the basis of the recall being in the same language as the target word. Seven cue words occurred in the recall results, and these were discarded from subsequent analysis.

The proportion of correctly recalled words for each study condition is given in Table 1 . An analysis of variance (ANOVA) was performed on the number of words correctly recalled. Throughout this paper, $M S_{\mathrm{e}}$ values are expressed as squared proportions. For the recall measure, the main effect of language of targets was not significant $(F<1)$, whereas the main effect of study conditions was significant $\left[F(2,80)=23.497, M S_{e}=0.018, p<.001\right]$. The interaction between language and study conditions failed to attain significance $(F<1)$. Tukey's $H S D$ procedures applied to the study-condition means revealed that the differences between both of the read conditions and the generation condition were reliable $(p<.01)$, but the difference between the repetition and translation conditions was not reliable $(p>.1)$.

The proportions of correct recognitions are also shown in Table 1. An ANOVA ${ }^{2}$ of the number of correct recognitions revealed a pattern of effects similar to that for recall. The main effect of language was not significant $(F<1)$, whereas the effect of conditions was significant $\left[F(2,78)=56.773, M S_{\mathrm{e}}=0.025, p<.001\right]$. The lan-

Table 1

Proportion of Correct Recall, Recognition, and False Alarms in Experiment 1

\begin{tabular}{lcccc}
\hline & \multicolumn{4}{c}{ Task } \\
\cline { 2 - 5 } & \multicolumn{2}{c}{ Recall } & \multicolumn{2}{c}{ Recognition } \\
\cline { 2 - 5 } Study Conditions & English & French & English & French \\
\hline Repetition & .08 & .11 & .52 & .44 \\
Translation & .13 & .14 & .53 & .57 \\
Generation & .29 & .28 & .85 & .78 \\
False alarms & - & - & .20 & .13 \\
\hline
\end{tabular}


guage and conditions interaction was not significant $(p>$ .1). Tukey's procedures revealed that the differences between both of the read conditions and the generation condition were reliable $(p<.01)$, whereas the difference between the two read conditions was not $(p>1)$. The false-alarm rates, shown in Table 1 , did not reliably differ as a function of target language $[t(39)=1.82, p>.07]$.

\section{Discussion}

The results of Experiment 1 demonstrated that a translation-based generation effect can occur in free recall, contrary to earlier reports (Durgunoglu \& Roediger, 1987; Slamecka \& Katsaiti, 1987). Although caution must be taken in the interpretation of the recognition results because they were obtained following the free-recall test and thus may have been influenced by the recall procedure, they also revealed a translation-based generation effect. The successful demonstration of a translation-based generation effect in Experiment 1 suggests that the subject selection and/or procedural factors discussed in the introduction may have been responsible for the earlier failures using within-subject designs. In particular, it is important to note that the earlier studies used intentional learning procedures whereas the present experiment used an incidental learning procedure.

McDaniel, Waddill, and Einstein (1968) obtained a between-subject generation effect using both category and semantic cues with measures of recall and recognition when subjects had not been informed of the retention tests prior to study (Experiment 2). However, when the subjects had been informed of the retention tests prior to the study phase (Experiment 3), the generation effect was abolished on both measures. Thus, it is possible that incidental learning is an important condition for the emergence of a translation-based generation effect. Experiment 2 included a comparison allowing for a test of this possibility in a between-subject design.

It has recently been reported that if the generating and reading study conditions are compared between lists, as opposed to within lists, generation effects can be substantially reduced (Hirshman \& Bjork, 1988) and even, in some cases, entirely eliminated (Begg \& Snider, 1987; Slamecka \& Katsaiti, 1987). These findings have led to two hypotheses that, for all intents and purposes, interpret the generation effect as an artifact of the within-list procedure.

First, Slamecka and Katsaiti (1987) argued, following Underwood (1983), that in within-list designs, generation effects may be caused by selective displaced rehearsal. That is, subjects may identify the independent variable and choose to devote more study time or effort to a particular level of it that attracts their attention. If it is assumed that subjects choose to devote greater time or effort to the generate conditions than to the read conditions, generation, rather than reading, could be expected to benefit from more rehearsal, thus leading to better retention.

The second interpretation (Begg \& Snider, 1987) leads to the argument that generation encoding causes subjects to adopt a criterion of simple identifiability in the generation condition. This generalizes to the reading-based condition, which in turn fosters less elaborate reading-based encoding than would otherwise be the case. It seems reasonable to suppose that such alterations in subjects' behavior due to the interaction of conditions would be more likely under incidental procedures than under intentional procedures. Thus, it is possible that the obtained generation effect in Experiment 1, using incidental study and within-subject comparisons, might be due to the operation of either or both of the above factors that were not present, or at least less influential, in the earlier bilingual studies that used intentional study procedures.

To directly test these accounts of the observed translationbased generation effect, Experiment 2 was performed using many of the same materials and study conditions that were employed in Experiment 1 but permitting between-subject comparisons of the conditions. Neither the selective displaced rehearsal nor the inhibition of reading process would be expected to influence betweensubject results, since each subject is only exposed to a single study condition.

To explore the importance of incidental versus intentional study conditions, the subjects in Experiment 2 studied two lists, the first under incidental study conditions and the second, following the retention tests for the first, under intentional study conditions. Although the influence of the first procedure on the second makes the results of such a comparison tenuous, it was felt that useful information might be provided that would not compromise the integrity of the incidental learning results.

\section{EXPERIMENT 2}

\section{Method}

\section{Subjects}

The subjects in Experiment 2 were 60 French-English bilinguals. They were selected in the same fashion and from a similar pool as were the subjects in Experiment 1. The subjects' language profiles were the same as in Experiment 1.

\section{Materials}

We obtained 120 pairs of French-English translation equivalents by using the same procedures followed in Experiment 1. Many of the pairs overlapped between the two studies, but they were independently chosen. Once again, half the words served as cues and targets in the study conditions, and the remainder served as distractors in the recognition tests. Four different versions of each type of list were prepared. Each list consisted of 30 pairs. Cues and targets were blocked into six sets of 30 French-English pairs. One third of the lists consisted of identical, same-language cue-target pairs, half in English and half in French. A second third consisted of translation pairs as cues and targets, with half of the targets in English and half in French. The final third consisted of 30 cues followed by the first letter of their translations and dashes for each letter in the target, with half of the targets in English and half in French. This resulted in 12 lists, each of which was employed the same number of times in the incidental and intentional learning procedures.

For each study list, separate recognition lists were prepared, six in French and six in English, consisting of all the targets presented in the relevant study condition and 30 distractors selected from the unused words in the same language as the targets. 
Lists were presented in the form of booklets consisting of an instruction page explaining the subjects' task and the 30 cue-target pairs on separate pages, as in Experiment 1 . The order of presentation of the pairs was randomized within lists.

\section{Procedure and Design}

Incidental learning. In the incidental learning procedure, 10 subjects were randomly assigned to each of the six groups defined by the crossing of the two independent variables. The first variable was the language of the targets. Half the subjects studied lists with English targets, and the remainder studied lists with French targets. The second variable consisted of the three study conditions. In the repetition condition, the subjects read the same word twice and then copied it. In the translation condition, the subjects read English and French translation equivalents and then copied the second (target) word. And in the generation condition, the subjects read the cue word and wrote out the opposite-language translation beginning with the letter provided and consisting of the number of letters indicated by the dashes. The subjects were run in small groups, as in Experiment 1. Oral communication with the subjects was done in French, but the written instructions were in the language corresponding to the subject's study condition.

Upon arrival in the test room, the subjects were told that the experimenter was interested in developing and refining material for use in studies of the linguistic abilities of bilinguals. No mention was made of any retention tests. The subjects were told to read the instructions on the first page of the booklet and were asked if they had any questions. The experimenter then told the subjects that they would have $8 \mathrm{sec}$, signaled by the experimenter, to complete each pair and that on the signal, they should turn to the next page. Following completion of the study phase, the subjects were asked to cross out threes, fives, and sevens in a table of random numbers for $30 \mathrm{sec}$. Following this, they were asked to write down as many of the targets as they could, in any order, on blank pieces of paper. The subjects were allowed $10 \mathrm{~min}$ for recall, following which they were given the recognition task.

Intentional learning. Following completion of the first recognition test, the subjects were informed that they would be asked to study a second list that would be followed by the same kinds of retention tests. The interval between the tests was kept as close to $5 \mathrm{~min}$ as possible. Each subject was assigned to the same condition in which he or she had been tested under incidental conditions; however, each subject received a different study list. The procedure was subsequently the same as that followed in the incidental learning procedure.

\section{Results}

Only five errors and failures of target generation occurred, and these items were discarded. For all conditions, the first three items of the study list were treated as a primacy buffer and excluded from subsequent analyses. Only target words recalled in the correct language were scored; in fact, no cue words were given as responses. An ANOVA was performed on the number of items correctly recalled. The recognition scores were converted to $d^{\prime}$ values and were subjected to an ANOVA along with the number of hits and false alarms.

Incidental learning. The means of the dependent variables for each study condition are presented in Table 2 . In general, retention was superior for the generation conditions than it was for the read conditions, whereas the read conditions did not differ from each other.

For recall, the main effect of the language of targets was not significant $(F<1)$, whereas the main effect of
Table 2

Recall and Recognition Measures as a Function of Encoding Conditions, Intentionality, and Language of Targets (Experiment 2)

\begin{tabular}{|c|c|c|c|c|c|c|}
\hline \multirow[b]{3}{*}{ Measures } & \multicolumn{6}{|c|}{ Encoding Conditions } \\
\hline & \multicolumn{2}{|c|}{ Repetition } & \multicolumn{2}{|c|}{ Translation } & \multicolumn{2}{|c|}{ Generation } \\
\hline & English & French & English & French & English & French \\
\hline \multicolumn{7}{|c|}{ Incidental Learning } \\
\hline Recall & .25 & .22 & .24 & .27 & .35 & .39 \\
\hline$d^{\prime}$ & 1.75 & 1.65 & 1.93 & 1.98 & 2.97 & 3.45 \\
\hline Hits & .65 & .55 & .64 & .61 & .81 & .86 \\
\hline False alarms & .17 & .20 & .22 & .15 & .16 & .08 \\
\hline \multicolumn{7}{|c|}{ Intentional Learning } \\
\hline Recall & .51 & .48 & .40 & .38 & .38 & .38 \\
\hline$d^{\prime}$ & 2.62 & 2.70 & 2.37 & 2.50 & 2.43 & 2.93 \\
\hline Hits & .80 & .83 & .82 & .81 & .80 & .86 \\
\hline False alarms & .07 & .09 & .12 & .11 & .08 & .03 \\
\hline
\end{tabular}

study condition was significant $[F(2,54)=12.343$, $\left.M S_{\mathrm{e}}=0.009, p<.001\right]$. The interaction between language of targets and study conditions failed to reach an acceptable level of significance $(F<1)$. Tukey's procedures performed on the means for the study conditions revealed reliable differences between the generation and translation conditions and between the generation and repetition conditions $(p<.01)$ but not between the translation and repetition conditions.

The $d^{\prime}$ analysis revealed a similar pattern. Language of targets was not significant $(F<1)$, whereas study conditions were significant $\left[F(2,54)=31.828, M S_{\mathrm{e}}=\right.$ $0.412, p<.001]$. Their interaction failed to reach an acceptable significance level $(p>.44)$.

Tukey's procedures applied to the study condition means also revealed reliable differences between the generation condition and both the translation and repetition conditions $(p<.01)$, whereas the two read conditions did not produce reliable differences.

Correct recognition and false-alarm proportions both produced a significant main effect of conditions $\left[F(2,54)=11.783, M S_{\mathrm{c}}=0.028, p<.001\right.$, and $F(2,54)=7.992, M S_{\mathrm{e}}=0.005, p<.001$, respectively]. Tukey's procedures revealed that the generation condition mean correct recognition proportions significantly exceeded both the translation condition mean and the repetition condition mean $(p<.01)$, whereas the generation condition false-alarm proportions were significantly lower than the repetition $(p<.05)$ and translation $(p<.01)$ proportions.

Intentional learning. The results of the intentional learning procedure, also shown in Table 2 , differ markedly from those with the incidental learning procedures because there is no evidence of greater retention in the generation conditions than in the read conditions. However, the results with the recall measure revealed a significant conditions effect $\left[F(2,54)=4.382, M S_{\mathrm{e}}=\right.$ $0.019, p<.02$ ]. Neither the language effect nor the interaction approached significance $(F<1)$. Tukey's procedures revealed that the significant effect was due to the 
fact that the repetition condition promoted greater recall than did either the translation or generation conditions $(p<.05)$. The recognition $d^{\prime}$ scores, correct recognition proportions, and false-alarm proportions were subjected to similar ANOVAs. None of the main effects or interactions achieved acceptable reliability. Only 10 instances of intrusions from the words studied under incidental procedures were observed.

\section{EXPERIMENT 3}

Experiment 2 revealed that a translation-based generation effect could be demonstrated in a between-subject design under incidental learning conditions but not under intentional learning conditions. However, the design of the study was such that, for all subjects, the incidental procedure preceded the intentional procedure, thus introducing the possibility that the intentional procedure results were influenced by the incidental procedure. Experiment 3 was performed to test for this possibility. Essentially, it replicated the procedures of the intentional learning phase of Experiment 2, but the subjects were never exposed to the incidental learning procedures.

\section{Method}

\section{Subjects}

The subjects were 60 French-English bilinguals who were selected in the same fashion as in the previous experiments. No subject participated in more than one of the experiments.

\section{Materials}

The materials were the same as those employed in Experiment 2, and similar booklets were prepared.

\section{Procedure}

The procedure was the same as that employed in the intentional learning phase of Experiment 2 . The subjects were informed prior to the study phase that their retention of the to-be-studied words would be tested, but they were not informed of the exact nature of the tests.

\section{Results}

As in the the previous studies, few errors or failures of generation occurred (nine in total). These were discarded from the calculations of proportions. The first three items of the study list were also excluded from analysis, as in Experiment 2. The ANOVAs performed on the num-

Table 3

Recall and Recognition Measures as a Function of Encoding Conditions and Language of Target for Experiment 3

\begin{tabular}{|c|c|c|c|c|c|c|}
\hline \multirow[b]{3}{*}{ Measures } & \multicolumn{6}{|c|}{ Encoding Conditions } \\
\hline & \multicolumn{2}{|c|}{ Repetition } & \multicolumn{2}{|c|}{ Translation } & \multicolumn{2}{|c|}{ Generation } \\
\hline & English & French & English & French & English & French \\
\hline Recall & .54 & .51 & .43 & .49 & .47 & .47 \\
\hline$d^{\prime}$ & 2.49 & 2.65 & 2.37 & 2.83 & 2.58 & 2.68 \\
\hline Hits & .81 & .85 & .83 & .86 & .88 & .84 \\
\hline False alarms & .07 & .09 & .08 & .12 & .12 & .07 \\
\hline
\end{tabular}

ber of correct recalls $\left(M S_{\mathrm{e}}=0.025\right), d^{\prime} \mathrm{s}\left(M S_{\mathrm{e}}=0.389\right)$, hits $\left(M S_{\mathrm{e}}=.007\right)$, and false alarms $\left(M S_{\mathrm{e}}=0.006\right)$ did not reveal any significant main effects or interactions. The means for the dependent variables are presented in Table 3.

\section{Discussion}

The results of Experiment 3 confirmed the absence of the translation-based generation effect under intentional learning procedures in the absence of a prior incidental procedure. The finding in Experiment 2 that, under intentional procedures, recall was significantly greater following the reading of repetitions than following either the reading or generation of translations was not replicated in Experiment 3, although a similar trend was observed at least for the English targets. The fact that the intentional procedures followed the incidental procedures in Experiment 2 may be responsible for this difference.

\section{GENERAL DISCUSSION}

The most important result of these experiments was the demonstration of the existence of a reliable translationbased generation effect using both free-recall and recognition measures of retention in relatively balanced bilingual subjects. The results of Experiment 2, showing that the generation effect occurred in a between-subject design, eliminates the possibility that it is an artifact of selective displaced rehearsal or within-list inhibition of reading. Although there are a number of methodological differences between the earlier failures to obtain a cross-language generation effect (Durgunoglu \& Roediger, 1967; Slamecka \& Katsaiti, 1987) and the present success, the absence of the effect under intentional learning conditions found in Experiments 2 and 3 suggests that this may be a crucial factor.

The second important finding was that no evidence was found in the present experiments for a retention superiority of the reading of translations over the reading of same-language repetitions. This is contrary to the predictions of dual-coding theory. This finding diverges from other reports that have found such retention superiority at least for short lags (Durgunoglu \& Roediger, 1987; Glanzer \& Duarte, 1971; Paivio et al., 1988). The most likely explanation of this discrepancy lies in the fact that in the present experiments, the subjects were instructed to recall only the targets, which were always in one language within any given condition, whereas in the Paivio et al. and Glanzer and Duarte studies, there were no targets as such, and recall was scored in terms of types. That is, credit was given for correct recall of a translation pair if either member was recalled. Similarly, Durgunoglu and Roediger encouraged their subjects to recall in either language (that of cues or targets) and only asked them to translate into the target language afterward. Although the recognition test list was entirely in English (in spite of the fact that some targets were in Spanish), subjects were encouraged to identify words as old regardless of the language in which they had seen it. 
These procedures clearly favor the dual-coding model, since subjects are actively encouraged to employ two strategies, either direct access to a remembered word or translation of a remembered word, in the memory tests for linguistically mixed materials (Slamecka \& Barlow, 1979). But they may not indicate that subjects actually retain more information following the study of translations than they do following the study of identical repetitions, only that bilingual subjects can translate between their two languages at retrieval. Subjects could have generated correct responses in the translation conditions without actually having stored the studied words. Under the present procedures, although the subjects could adopt the recall cue word and translate strategy, they were discouraged from doing so by the instructions. This appears to have been sufficient to abolish the read-translation superiority. The same result occurred in recognition when the subjects were only given the words in the target language and were not encouraged to translate at the time of the memory test.

From the perspective of the investigation of bilingual memory, the present results are more easily handled within the framework of a single-code model of bilingual linguistic representation (MacLeod, 1976; Potter et al., 1984) than within the framework of a dual-code model. Single-code models generally propose that translation equivalents access a common conceptual representation in an alinguistic semantic reference system. It follows from this that the processing of translations should produce effects similar to those obtained with same-language repetitions when processing implicates semantic factors. A single-code model could explain as easily as a dualcode model the superiority of translations over identical repetitions when subjects are encouraged to recall in either language, since it postulates two ways of accessing the common conceptual representation for linguistic equivalents and two possible output routes from the same representation. The single-code model would also suggest that processing effects due to such manipulations as generation should occur both intra- and interlanguage. Thus, it would anticipate that generation effects should not be confined to within-language manipulations.

From the perspective of the analysis of the generation effect, Experiment 1 adds a cross-language relationship to the long list of within-language relationships that can support a generation effect. The generation effect demonstrated in Experiment 2 adds to the much shorter list of those obtained in between-subject designs (Graf, 1982; McDaniel, Riegler, \& Waddill, 1990; McDaniel \& Waddill, 1990; McDaniel et al., 1988; Schmidt \& Cherry, 1989, Experiment 5; Slamecka \& Graf, 1978, Experiment 1). The absence of the generation effect in Experiments 2 and 3 under intentional learning conditions is similar to that found by McDaniel et al. (1988).

Perhaps more importantly, the way the generation effect was eliminated was similar in the present study and in that of McDaniel et al. (1988). Retention in the generation conditions did not show any major reductions, going from incidental to intentional procedures, but rather the recall and correct recognition proportions for the reading conditions increased. This suggests that what appears to be a generation effect is more likely a reflection of alterations in the information processing of subjects in the reading conditions. The generation effect may in fact not be due to enhanced processing of the generated items but rather to inhibitory processing of the read items, as suggested by Begg and Snider (1987). But the inhibition need not be due to the fact that the same subjects are also asked to generate. The suggestion made here, similar to that of McDaniel et al. (1988), is that generation effects will emerge when conditions lead to cursory or less distinctive (Begg, Snider, Foley, \& Goddard, 1989) processing of information in the read conditions and will disappear if either instructions or some other manipulation leads to enhanced processing of the read items.

McDaniel and his colleagues (McDaniel et al., 1990; McDaniel \& Waddill, 1990; McDaniel et al., 1988) have proposed that three factors may play a role in producing generation effects: (1) alterations in individual-item processing of targets, (2) relational processing between cues and targets, and (3) whole-list relational features. In the present experiments, uncategorized lists were used so that no whole-list relational elements were present to support generation effects. As discussed in the introduction, the connections between translation equivalents are generally considered to be relatively strong (witness also the extremely low number of translation failures reported in the present studies) and automatic (Guttentag, Haith, Goodman, \& Hauch, 1984). Thus, it seems unlikely that generation of translations would provoke an enhancement of such relational processing over simple reading of the translation. In fact, it is possible that the relational effects in reading may overshadow and thus inhibit individual-item processing. That is, in the reading conditions, with no intent to remember, subjects may concentrate on the identification (correctness of the translation) and thus treat the individual-item information rather superficially. Under generation conditions, the latter type of information would be more fully processed, since the subject must reconstruct the word that would facilitate subsequent recall and recognition. Under intentional learning procedures, individual-item processing of the target words would be expected to increase in the reading conditions relative to any changes in the generation conditions, thus reducing or eliminating any differences in retention.

With the exceptions of the Slamecka and Graf (1978), Schmidt and Cherry (1989, Experiment 5), and Graf (1982) studies that revealed between-subject generation effects in recognition memory, successful demonstrations of generation effects using between-subject procedures appear to have employed incidental procedures. However, the three exceptions all seem to have employed procedures that might be expected to reduce the processing of individual-item information in the reading groups.

Schmidt and Cherry (1989) did not follow the normal read-condition procedures. They asked their subjects to associate the cue-target pairs, which would emphasize relational processing, possibly at the expense of individual- 
item processing, and which would be expected to have a negative impact on recognition. They also observed a positive effect of generation under intentional learning conditions when subjects were asked to recall word pairs, but only when recall of targets alone was scored. However, this scoring method did not include the targets recalled when their cues were also recalled. When these are counted, the generation effect is not apparent.

The Graf (1982) procedure involved the reading of sentences, which would also probably emphasize relational factors at the expense of individual-item processing. The Slamecka and Graf (1978, Experiment 1) procedure combined five different relationships within conditions, which might also be expected to cause subjects to attend more to relational factors than to individual-item information and thus lead to reading-based retention deficits.

Also compatible with the present analysis of generation effects, Watkins and Sechler (1988, Experiment 2), using a within-subject procedure designed to minimize the possibility of intralist effects in assessing generation versus reading effects, found that generation effects were substantially larger under incidental than under intentional procedures and that the decline under intentional procedures was almost entirely due to increased recall in the read conditions.

In summary, the present research has demonstrated that a robust translation-based generation effect can be demonstrated in bilingual subjects. However, the appearance of the effect depends on the presence of conditions that would inhibit the processing of individual-item information in the reading conditions.

\section{REFERENCES}

Arnedt, C. S., \& Gentile, J. R. (1986). A test of dual coding theory for bilingual memory. Canadian Journal of Psychology, 40, 290-299.

BEGG, I., \& SNIDER, A. (1987). The generation effect: Evidence for generalized inhibition. Journal of Experimental Psychology: Learning, Memory, \& Cognition, 13, 553-563.

BegG, I., SNider, A., Foley, F., \& Goddard, R. (1989). The generation effect is no artifact: Generating makes words distinctive. Journal of Experimental Psychology: Learning, Memory, \& Cognition, 15, 977-989.

DALRYMPLE-AlFord, E. C. (1985). Language switching during bilingual reading. British Journal of Psychology, 76, 111-122.

Durgunoglu, A. Y., \& Roediger, H. L., III (1987). Test differences in accessing bilingual memory. Journal of Memory \& Language, 26, 377-391.

Glanzer, M., \& Duarte, A. (1971). Repetition between and within languages in free recall. Jourmal of Verbal Learning \& Verbal Behavior, 10, 625-630.

GrAF, P. (1982). The memorial consequences of generation and transformation. Journal of Verbal Learning \& Verbal Behavior, 21, 539-548.

Gutrentag, R. E., Haith, M. M., Goodman, G. S., \& Hauch, J. (1984). Semantic processing of unattended words by bilinguals: A test of the input switch mechanism. Journal of Verbal Learning \& Verbal Behavior, 23, 178-188.

Hirshman, E., \& Bjork, R. A. (1988). The generation effect: Support for a two-factor theory. Journal of Experimental Psychology: Learning, Memory, \& Cognition, 14, 484-494.

Kolers, P. A., \& Gonzalez, E. (1980). Memory for words, synonyms, and translations. Journal of Experimental Psychology: Human Learning \& Memory, 6, 53-65.
LiepmanN, D., \& SAEGERT, J. (1974). Language tagging in bilingual free recall. Journal of Experimental Psychology, 103, 1137-1141.

MACLEOD, C. M. (1976), Bilingual episodic memory: Acquisition and forgetting. Journal of Verbal Learning \& Verbal Behavior, 15, 347-364.

MaCnamara, J. (1967). How can one measure the extent of a person's bilingual proficiency? In L. G. Kelly (Ed.), Description and measurement of bilingualism: An international seminar (pp. 79-97). Toronto: University of Toronto Press.

McDaniel, M. A., Riegler, G. L., \& Waddill, P. J. (1990). Generation effects in free recall: Further support for a three-factor theory. Joumal of Experimental Psychology: Leaming, Memory, \& Language, 16, 789-798

MCDANiEl, M. A., \& Waddill, P. J. (1990). Generation effects for context words: Implications for item-specific and multifactor theories. Joumal of Memory \& Language, 29, 201-211.

McDaniel, M. A., Waddill, P. J., \& Einstein, G. O. (1988). A contextual account of the generation effect: A three-factor theory. Journal of Memory \& Language, 27, 521-536.

Paivio, A. (1986). Mental representation: A dual-coding approach. New York: Oxford University Press.

Paivio, A., Clark, J. M., \& Lambert, W. E. (1988). Bilingual dualcoding theory and semantic repetition effects on recall. Journal of Experimental Psychology: Learning, Memory, \& Cognition, 14, 163-172.

Paivio, A., \& Desrochers, A. (1980). A dual-coding approach to bilingual memory. Canadian Journal of Psychology, 34, 388-399.

Paivio, A., \& Lambert, W. E. (1981). Dual coding and bilingual memory. Journal of Verbal Learning \& Verbal Behavior, 20, 532-539.

Potter, M. C., So, K.-F., von Eckardt, B., Feldman, L. B. (1984). Lexical and conceptual representation in beginning and proficient bilinguals. Joumal of Verbal Leaming \& Verbal Behavior, 23, 23-38.

SCHmidT, S. R., \& Cherry, K. (1989). The negative generation effect: Delineation of a phenomenon. Memory \& Cognition, 17, 359-369.

SChwanenflugel, P. J., \& Rey, M. (1986). Interlingual semantic facilitation: Evidence for a common representational system in the bilingual lexicon. Joumal of Memory \& Language, 25, 605-618.

Slamecka, N. J., \& Barlow, W. (1979). The role of semantic and surface features in word repetition effects. Journal of Verbal Learning \& Verbal Behavior, 18, 617-627.

Slamecka, N. J., \& Graf, P. (1978). The generation effect: Delineation of a phenomenon. Journal of Experimental Psychology: Human Learning \& Memory, 4, 592-604.

Slamecka, N. J., \& Katsaiti, L. T. (1987). The generation effect as an artifact of selective displaced rehearsal. Journal of Memory \& Language, 26, 589-607.

UNDERWOOD, B. J. (1983). Attributes of memory. Glenview, IL: Scott, Foresman.

VAID, J. (1988). Bilingual memory representation: A further test of dual coding theory. Canadian Journal of Psychology, 42, 84-90.

VIKIS-FreIbergs, V. (1974). Fréquence d'usage des mots au Québec. Montréal: Les Presses de l'Université de Montréal.

WATKINS, M. J., \& SeChLER, E. S. (1988). Generation effect with an incidental memorization procedure. Journal of Memory \& Language, $27,537-544$.

\section{NOTES}

1. Curiously, the recall results were taken as support for the existence of a generation effect. The authors pointed out this discrepancy in a footnote. However, they interpreted their data as if a translationbased generation effect had occurred for recall but not for recognition or word-fragment completion. It would have seemed more prudent to argue that the translation-based generation effect was absent for all three tasks.

2. One subject was eliminated for failure to correctly perform the recognition task.

(Manuscript received July 5, 1991; revision accepted for publication December 9, 1992.) 